\title{
Determination of Midodrine in Human Plasma by High-Performance Liquid Chromatography with Fluorescence Detection
}

\author{
Hideyuki Yoshida, Yuki OHno, Kenichiro YoshiKuni, Kenichiro TodoroKI, Hitoshi NOHTA, \\ and Masatoshi YAMAGUCHI ${ }^{\dagger}$
}

\author{
Faculty of Pharmaceutical Sciences, Fukuoka University, Nanakuma, Johnan, Fukuoka 814-0180, Japan
}

\begin{abstract}
A simple and sensitive fluorometric high-performance liquid chromatographic method was developed for the determination of midodrine in human plasma. After liquid-liquid extraction from plasma, the drug and 2-phenylglycinol (internal standard) were converted into the corresponding fluorescent derivatives by reaction with 3,4-dihydro-6,7dimethoxy-4-methyl-3-oxoquinoxaline-2-carbonyl chloride, a fluorescence derivatization reagent for amines. The derivatives were separated within $30 \mathrm{~min}$ on a reversed-phase column using isocratic elution with acetonitrile-methanol-water $(10: 30: 60, \mathrm{v} / \mathrm{v})$ and were detected spectrofluorometrically at $485 \mathrm{~nm}$ with excitation at 400 $\mathrm{nm}$. The detection limit for midodrine was $0.3 \mathrm{pmol}(76 \mathrm{pg})$ per $\mathrm{mL}$ plasma at a signal-to-noise ratio of 3 .
\end{abstract}

(Received October 10, 2002; Accepted November 15, 2002)

Midodrine,

$( \pm)-1-\left(2^{\prime}, 5^{\prime}\right.$-dimethoxyphenyl)-2-glycinamidoethanol, is a long acting $\alpha$-adrenergic stimulating agent. Midodrine causes elevation of systemic blood pressure accompanied by a reduction in heart rate. ${ }^{1-3}$

In order to determine the plasma level of midodrine, several methods based on radioisotopic labeling, ${ }^{4}$ gas chromatography (GC), ${ }^{5}$ and high-performance liquid chromatography (HPLC) with native fluorescence detection ${ }^{6}$ and with UV detection ${ }^{7}$ have been developed. However, the GC method is not simple and needs a tedious clean-up procedure, and the native fluorescence HPLC method is not also simple and needs a sophisticated column-switching technique. Although the HPLC-UV detection can supply an enantioseparation of midodrine racemate in the same run using human $\alpha_{1}$-acid glycoprotein as a chiral stationary phase of the HPLC column, it does not have enough sensitivity for clinical use. Hence, a simple, reproducible and sensitive assay for midodrine in plasma is needed.

We have previously developed a highly sensitive fluorometric HPLC method for the determination of primary and secondary amines. ${ }^{8}$ The method is based on the derivatization reaction of the amines with 3,4-dihydro-6,7-dimethoxy-4-methyl-3oxoquinoxaline-2-carbonyl chloride (DMEQ-COCl), a sensitive and selective fluorescence derivatization reagent for amines. The HPLC method with DMEQ-COCl has been successfully applied to the determination of biologically important amines, $\beta$-phenylethylamine in human plasma $^{9}$ and $1,2,3,4-$ tetrahydroisoquinoline in rat brain,,$^{10}$ and of a drug, amantadine, in human plasma. ${ }^{11} \mathrm{We}$ found that midodrine, which has a primary amino group, reacted with DMEQ-COCl to give a highly fluorescent derivative (Fig. 1).

The purpose of the present research is to develop a simple, sensitive and reproducible HPLC method using DMEQ-COCl for the quantification of midodrine in human plasma. 2Phenylglycinol (PG) was used as an internal standard (IS) for

† To whom correspondence should be addressed.

E-mail: masayama@fukuoka-u.ac.jp the precise determination. The plasma concentration level of midodrine from a healthy male volunteer after oral administration of midodrine hydrochloride was measured by the established method.

\section{Experimental}

\section{Chemicals and solutions}

All chemicals and solvents were of the highest purity available and were used as received. Distilled water, purified with a Milli-QII system (Millipore, Milford, MA, USA), was used for all aqueous solutions. Midodrine hydrochloride was obtained from ICN Biomedicals (Irvine, CA, USA). PG was purchased from Tokyo Kasei Kogyo (Tokyo, Japan). DMEQ$\mathrm{COCl}$ was obtained from Dojindo Laboratories (Kumamoto, Japan). ${ }^{12}$

Stock solutions (10 mM each) of midodrine and PG were prepared in water, stored at $4^{\circ} \mathrm{C}$, and then further diluted with water to the desired concentrations before use. DMEQ-COCl solution $(2.0 \mathrm{mM})$ dissolved in acetonitrile was used within a day.

\section{Plasma samples}

A healthy volunteer (male, 22 years old, $65 \mathrm{~kg}$ ) in our laboratory took $6 \mathrm{mg}$ of midodrine hydrochloride orally at 9 a.m. after an overnight fasting. He understood the purpose and importance of the experiments, and took the drug of his own free will. After administration of the drug, blood samples were collected to heparinized tubes at $0,0.5,1,2,4$, and $6 \mathrm{~h}$, and the heparinized-plasmas, obtained by centrifugation at $1000 \mathrm{~g}$ for 10 min at $4^{\circ} \mathrm{C}$, were used as the samples. In the same manner, drug-free pooled plasma samples were obtained from five healthy volunteers. The plasma samples were immediately frozen and stored at $-20^{\circ} \mathrm{C}$ until assay.

\section{Pretreatment procedure}

A $0.5-\mathrm{mL}$ aliquot of plasma was pipetted into a $10-\mathrm{mL}$ screw- 

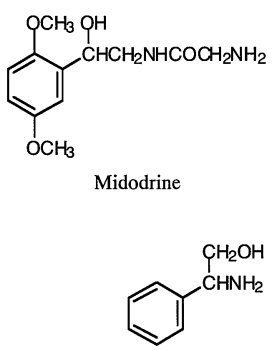

2-Phenylglycinol (IS)
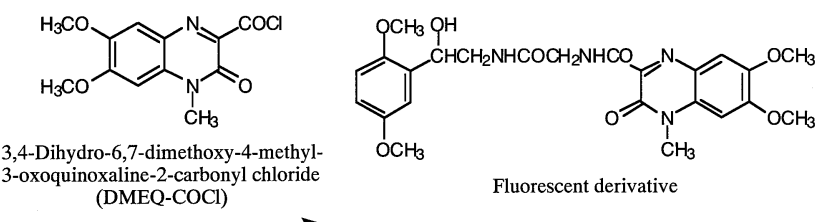

Fluorescent derivative

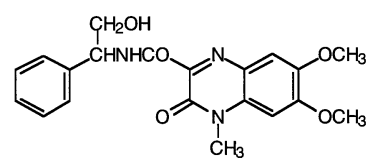

Fluorescent derivative

Fig. 1 Fluorescence derivatization of midodrine and 2-phenylglycinol (IS) with DMEQ-COCl.

capped test tube together with $10 \mu \mathrm{L}$ of the IS solution $(1.0$ $\mathrm{nmol} / \mathrm{mL} \mathrm{PG}), 20 \mu \mathrm{L}$ of $1.0 \mathrm{M}$ hydrochloric acid and $3 \mathrm{~mL}$ of ethyl acetate. After vortex-mixing for $c a .5 \mathrm{~min}$, the mixture was centrifuged at $1000 \mathrm{~g}$ for $10 \mathrm{~min}$. The upper layer was aspirated and discarded, and $20 \mu \mathrm{L}$ of $2.0 \mathrm{M}$ sodium hydroxide and $3 \mathrm{~mL}$ of ethyl acetate were added. After being extracted in the same manner, the organic layer $(2.5 \mathrm{~mL})$ was transferred to 5-mL polypropylene test tube, and was evaporated to dryness under a nitrogen stream. The residue, dissolved in $300 \mu \mathrm{L}$ of water, was subjected to the following derivatization procedure as a plasma extract.

\section{Derivatization procedure}

To a $300-\mu \mathrm{L}$ aliquot of a standard solution (or plasma extract) placed in a $5-\mathrm{mL}$ polypropylene test tube, were added $100 \mu \mathrm{L}$ of $2.0 \mathrm{mM}$ DMEQ-COCl and $20 \mu \mathrm{L}$ of $0.1 \mathrm{M}$ potassium carbonate. After vortex-mixing for $c a .1 \mathrm{~min}$ at room temperature, $20 \mu \mathrm{L}$ of $0.1 \mathrm{M}$ hydrochloric acid was added to stop the derivatization reaction, and a $50-\mu \mathrm{L}$ portion of the resulting mixture was injected into the chromatograph. To prepare the reagent blank, a $300-\mu \mathrm{L}$ volume of water in place of the standard solution was subjected to the same procedure.

\section{Apparatus and HPLC conditions}

An isocratic LC system consisted of a Hitachi (Tokyo, Japan) L-7100 liquid chromatograph pump, a Rheodyne (Cotati, CA, USA) Model 7125 syringe-loading sample injector equipped with a $20-\mu \mathrm{L}$ sample loop, a reversed-phase TSKgel ODS-120T column $(250 \times 4.6 \mathrm{~mm}$ i.d., particle size $5 \mu \mathrm{m}$; Tosoh, Tokyo, Japan), and a Hitachi L-7480 spectrofluorometer fitted with a $12-\mu \mathrm{L}$ flow-cell. A mixture of acetonitrile-methanol-water $(10: 30: 60, v / v)$ was used as a mobile phase. The flow-rate of the mobile phase was set at $1.0 \mathrm{~mL} / \mathrm{min}$, and the column temperature was ambient $\left(23 \pm 3^{\circ} \mathrm{C}\right)$. The fluorescence detector was operated at the excitation and emission wavelengths of 400 $\mathrm{nm}$ and $485 \mathrm{~nm}$, respectively, and the slit-widths of both the monochromators were set at $15 \mathrm{~nm}$.

Uncorrected fluorescence excitation and emission spectra of the eluates were measured with a Hitachi F-3010 fluorescence spectrophotometer in $10 \times 10-\mathrm{mm}$ quartz cell; spectral bandwidths of $5 \mathrm{~nm}$ were used for both excitation and emission monochromators.

\section{Results and Discussion}

\section{HPLC conditions}

Baseline separation of the DMEQ derivatives of midodrine and PG was achieved using a reversed-phase column, TSKgel ODS-120T, and acetonitrile-methanol-water (10:30:60, v/v) as
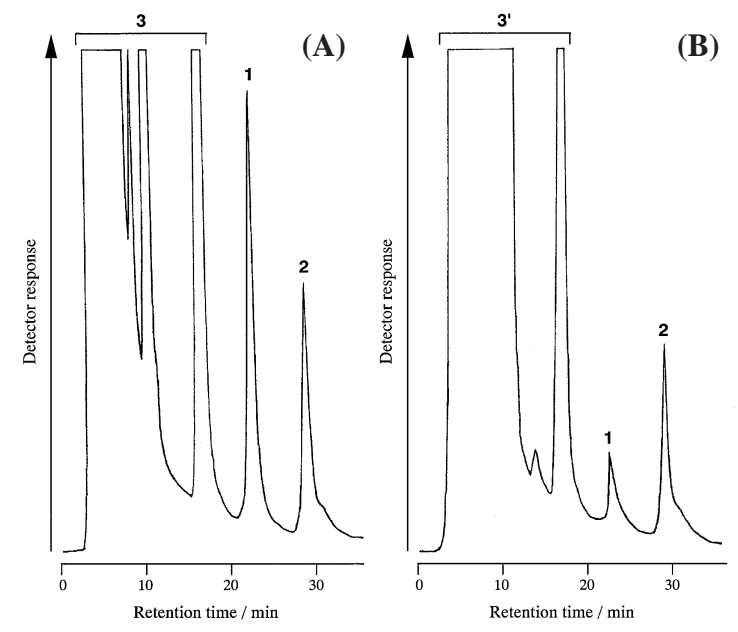

Fig. 2 Chromatograms obtained with (A) a standard solution and (B) a plasma spiked with midodrine. Peaks: 1, midodrine; 2, PG (IS); 3 , reagent blank components; 3', reagent blank components and plasma endogenous substances. A standard mixture of midodrine and PG (5.1 and 1.0 pmol on column, respectively) and a plasma extract (20 pmol midodrine per $\mathrm{mL}$ plasma; $0.75 \mathrm{pmol}$ on column) were treated as in the procedure.

the eluent. Figure 2 (A) shows a typical chromatogram obtained with a standard mixture of midodrine and PG. They gave the respective single peaks in the chromatogram. The DMEQ derivatives of these amines in the mobile phase showed the same fluorescence excitation (maximum, $400 \mathrm{~nm}$ ) and emission (maximum, $485 \mathrm{~nm}$ ) spectra.

Some biogenic amines ( $p$ - and $m$-tyramine, histamine, octopamine, catecholamines, amino acids, and some polyamines) reacted with $\mathrm{DMEQ}-\mathrm{COCl}$ to give the respective fluorescent derivatives. However, these amines were co-eluted with DMEQ-COCl and the degradation products of DMEQ$\mathrm{COCl}$ (peaks 3 in Fig. 2), or were not eluted from the column under the HPLC conditions used, and did not interfere with the determination of midodrine.

\section{Derivatization conditions}

Acetonitrile as a solvent for the derivatization reaction provides the most intense peaks for midodrine and PG; acetone, $\mathrm{N}, \mathrm{N}$-dimethylformamide, and methanol gave smaller peaks (about $50-90 \%$ of those obtained in acetonitrile). Acetonitrile was therefore chosen for the recommended procedure. DMEQ$\mathrm{COCl}$ gave the most intense and constant peaks for the two compounds at concentrations greater than $1.5 \mathrm{mM}$; a concentration of $2.0 \mathrm{mM}$ was selected. Potassium carbonate 
was used to facilitate the derivatization of midodrine and PG with DMEQ-COCl. The peak heights were maximal when the concentration of potassium carbonate was in the range of 0.05 $0.15 \mathrm{M} ; 0.1 \mathrm{M}$ was selected as optimum.

The derivatization reaction of midodrine and PG with DMEQ$\mathrm{COCl}$ proceeded rapidly and independently of the temperature $\left(0-50^{\circ} \mathrm{C}\right)$; both peaks for the compounds reached maximal heights within $15 \mathrm{~s}$ even at $0^{\circ} \mathrm{C}$. Therefore, vortex-mixing for ca. $1 \mathrm{~min}$ at room temperature was enough to complete the derivatization reaction. The DMEQ-labeled derivatives of the two compounds in the final mixture were stable, and still gave the constant fluorescence intensities after standing for at least 1 $\mathrm{h}$ in the dark at $50^{\circ} \mathrm{C}$ and for $8 \mathrm{~h}$ in the daylight at room temperature.

\section{Pretreatment of plasma samples}

Some pretreatment methods, such as deproteinization using several acids or organic solvents, solid-phase extraction, and liquid-liquid extraction, have been commonly available. Among them, the two-step liquid-liquid extraction gave the best result; the first step was to remove the interfering substances and the second step was to concentrate the amines. Midodrine and PG were extracted effectively under the two-step extraction conditions with ethyl acetate from plasma, as described in Experimental. Figure 2 (B) shows a chromatogram obtained with the plasma spiked with midodrine and PG.

The recoveries (mean \pm standard deviation) of midodrine and PG spiked in pooled normal human plasma (20 pmol each per $\mathrm{mL}$ plasma) were $65.8 \pm 2.8 \%$ and $68.3 \pm 3.8 \%$, respectively.

\section{Validation of the method}

The detection limits (fmol on column, signal-to-noise ratio $=$ 3) were 56 and 11 for midodrine and PG, respectively.

The determination limit for midodrine was $0.3 \mathrm{pmol}(76 \mathrm{pg})$ per $\mathrm{mL}$ plasma. This sensitivity is nearly equal to that of the previous GC $^{5}$ and HPLC methods. ${ }^{6,7}$ The inter-day precision was established by repeated determination $(n=5)$ of drug concentration on plasma spiked with midodrine $(20$ pmol per $\mathrm{mL}$ ); the relative standard deviation was $4.5 \%$.

A good linear relationship was observed between the ratio of the peak height of midodrine to that of PG and the amount of midodrine added to plasma up to at least $300 \mathrm{pmol}$ per $\mathrm{mL}$ plasma; the linear correlation coefficient $(n=6)$ was 0.9998 . Moreover, the slope of the calibration graph for midodrine did not change with the plasma used. These results show that the present internal standard method permits the precise determination of midodrine in plasma samples.

\section{Determination of midodrine in human plasma}

In Fig. 2 (B), peaks 1 and 2 were identified as the DMEQ derivatives of midodrine and $\mathrm{PG}$, respectively, on the basis of their retention times in comparison with those of the standard compounds, and by co-chromatography of the standard and the sample with several combinations of various percentages of acetonitrile and methanol as mobile phase. The fluorescence excitation (maximum, $400 \mathrm{~nm}$ ) and emission (maximum, 485 $\mathrm{nm})$ spectra of the eluates of the peaks in Fig. 2 (B) were in good agreement with those for pure compounds (Fig. 2 (A)).

In order to evaluate the proposed method for clinical use, it was applied to the monitoring of midodrine in human plasma sample obtained after a single oral dose of midodrine hydrochloride $(6 \mathrm{mg}$ administration to male, 22 years old, 65 $\mathrm{kg}$ ). Figure 3 shows the time-concentration curve obtained by the plasma sample. The concentration of midodrine reached a maximal at $0.5 \mathrm{~h}$ after oral administration and then decreased

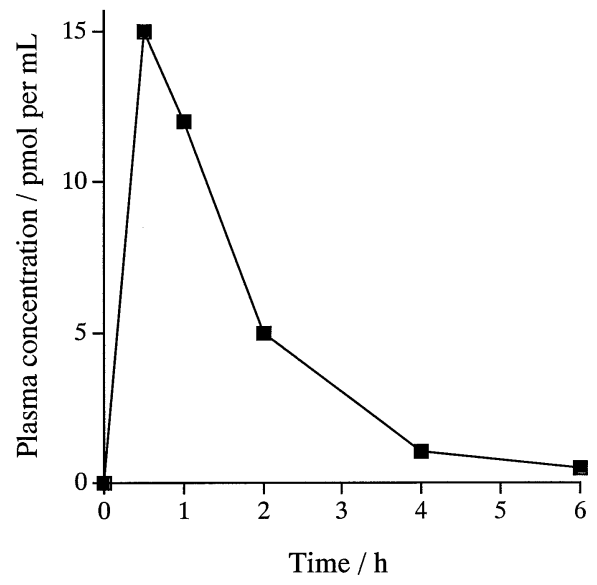

Fig. 3 Concentration of midodrine in plasma after oral administration of midodrine hydrochloride $(6 \mathrm{mg})$ to a healthy volunteer (male, 22 years old, $65 \mathrm{~kg}$ ).

quickly. The pattern of the curve was almost identical to those obtained by other workers. ${ }^{5,6}$

\section{Conclusion}

The present fluorometric HPLC method using DMEQ-COCl needs only two-step liquid-liquid extraction for the pretreatment of the plasma sample. Moreover, it offers higher sensitivity to permit the quantification of midodrine in $0.5 \mathrm{~mL}$ of human plasma after oral administration of midodrine hydrochloride. The method can thus be useful in therapeutic and pharmaceutical investigations of midodrine.

\section{Acknowledgements}

The authors are grateful to Mr. K. Ishimaru for his skilful assistance.

\section{References}

1. O. Thlesius, Int. Eur. J. Clin. Pharmacol., 1979, 16, 423.

2. H. Pittner, Gen. Pharmac., 1983, 14, 107.

3. D. Mc Tavish, Drugs, 1989, 38, 757.

4. N. Kolossa, W. G. Schutzenberger, H. Wiener, and P. Krivanek, Arch. Int. Pharmacodyn., 1979, 238, 96.

5. S. Tsutsumi, T. Suwa, N. Masuda, T. Minagawa, M. Sano, T. Tamatani, and K. Tsuchida, Clin. Report, 1987, 21, 1895.

6. W. Posch and W. Lindner, Biomed. Chromatogr., 1989, 3, 153.

7. M. G. Quagla, A. Farina, E. Bossú, and V. Cotichini, J. Pharm. Biomed. Anal., 1998, 18, 171.

8. J. Ishida, M. Yamaguchi, T. Iwata, and M. Nakamura, Anal. Chim. Acta, 1989, 223, 319.

9. J. Ishida, M. Yamaguchi, and M. Nakamura, Anal. Biochem., 1990, 184, 86.

10. J. Ishida, M. Yamaguchi, and M. Nakamura, Anal. Biochem., 1991, 195, 168.

11. T. Iwata, H. Fujino, J. Sonoda, and M. Yamaguchi, Anal. Sci., 1997, 13(supplement), 467.

12. T. Iwata, M. Yamaguchi, S. Hara, M. Nakamura, and Y. Ohkura, J. Chromatogr., 1986, 362, 209. 